\title{
Reconstructing Intra-Tumor Heterogeneity via Convex Optimization and Branch-and-Bound Search
}

\author{
Shorya Consul ${ }^{*}$ \\ The University of Texas at Austin \\ shoryaconsul@utexas.edu
}

\author{
Haris Vikalo \\ The University of Texas at Austin \\ hvikalo@ece.utexas.edu
}

\begin{abstract}
Studies of intra-tumor heterogeneity using high-throughput sequencing data, i.e., assembly of tumor populations and inference of their relative frequencies, may provide highly valuable information about molecular signatures of cancer and point towards specific therapeutic treatments. Reconstructing heterogeneous tumor populations, however, is a challenging task rendered difficult by complex mutations and further exacerbated by the fact that high-throughput sequencing reads are short relative to genomic regions experiencing structural mutations. In this paper, we present a novel algorithmic framework for computationally efficient and accurate analysis of tumor clonal populations. Given locations of copy number aberrations, the proposed method quantifies gains and losses of the affected regions, and determines relative frequencies of the populations in a clonal mixture. This is achieved by formulating the reconstruction as a mixed-integer optimization problem and solved via a combination of convex optimization and branch-and-bound search. The developed algorithm was tested on realistic synthetic as well as experimental data and shown to outperform state-of-the art competing methods in a variety of scenarios.
\end{abstract}

\section{CCS CONCEPTS}

- Applied computing $\rightarrow$ Computational genomics.

\section{KEYWORDS}

tumor heterogeneity, high-throughput sequencing, convex optimization, branch-and-bound search

\section{ACM Reference Format:}

Shorya Consul and Haris Vikalo. 2019. Reconstructing Intra-Tumor Heterogeneity via Convex Optimization and Branch-and-Bound Search. In 10th ACM International Conference on Bioinformatics, Computational Biology and Health Informatics (ACM-BCB '19), September 7-10, 2019, Niagara Falls, NY, USA. ACM, New York, NY, USA, 6 pages. https://doi.org/10.1145/3307339. 3342178

*Both authors contributed equally to this research.

Permission to make digital or hard copies of all or part of this work for personal or classroom use is granted without fee provided that copies are not made or distributed for profit or commercial advantage and that copies bear this notice and the full citation on the first page. Copyrights for components of this work owned by others than the author(s) must be honored. Abstracting with credit is permitted. To copy otherwise, or republish, to post on servers or to redistribute to lists, requires prior specific permission and/or a fee. Request permissions from permissions@acm.org.

ACM-BCB '19, September 7-10, 2019, Niagara Falls, NY, USA

(c) 2019 Copyright held by the owner/author(s). Publication rights licensed to ACM ACM ISBN 978-1-4503-6666-3/19/09 _\$15.00

https://doi.org/10.1145/3307339.3342178

\section{INTRODUCTION}

Cancer is a progressive disease associated with chromosomal aberrations that interfere with normal cellular processes and promote proliferation of abnormal cells. The onset and progression of cancer is in part driven by somatic mutations which, unlike inherited germline mutations, occur and accumulate over a lifetime of an individual [16]. Somatic mutations lead to a variety of genomic alterations, ranging from simple single nucleotide variations (SNVs) to more complex genomic rearrangements $[1,12,28,30]$. The latter include deletions and duplications of DNA segments that result in copy number aberrations (CNAs) $[11,17,32]$, as well as more complex structural variations such as inversions and translocations [2]. Deletions and duplications of DNA segments are illustrated in Fig. 1. Some genomic mutations and rearrangements impact cellular functions severely; examples include deletions of tumor suppressing genes and amplifications of cancer-promoting oncogenes [5, 14]. Alterations lead to diverse tumor genomes that for the same type of cancer, generally vary from one patient to another. Even a single tumor may consist of cells affected by different genomic alterations, a phenomenon referred to as intra-tumor heterogeneity $[9,27,29]$.

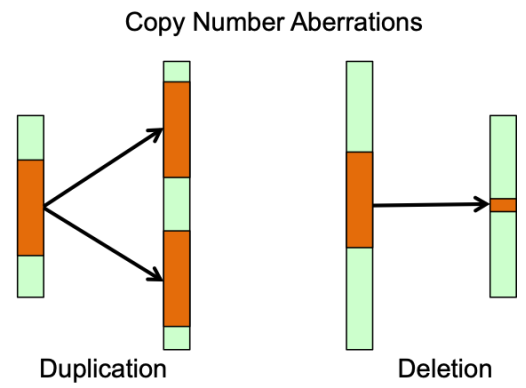

Figure 1: An illustration of duplications and deletions, common genomic aberrations in tumor cells.

Rapid advancements in DNA sequencing technology, facilitating faster as well as more affordable and accurate sequencing tasks, have enabled reconstruction of individual genetic blueprints and are thus paving the way towards personalized medicine [7, 8, 21]. Individually tailored medical treatments enabled by routine sequencing tests are expected to improve effectiveness of health care, including that of cancer patients. However, before the promise of personalized cancer treatment comes to fruition, we need to develop methods for accurate reconstruction of genomic mutations that characterize an individual's tumor cells. 
Inferring the composition of heterogeneous mixtures of tumor genomes in individual patients may provide highly valuable information about molecular signatures of cancer and point towards specific therapeutic treatments [3, 19, 24, 26]. Moreover, multi-sample studies of such heterogeneous mixtures could help identify somatic mutations associated with aggressive tumor growth $[6,10,15,22]$. Tumor populations are either clonal, if they inherited mutations from the common ancestor, or subclonal, if their mutations happened later in the development of the tumor. They may also potentially be polyclonal, if the origin of distinct populations can be traced back to different ancestors. A major challenge in somatic mutations analysis stems from the fact that the sample being tested typically contains both normal cells as well as the cells from one or more subclonal populations. Therefore, a fundamental computational task in this setting is to separate genomic material coming from normal cells from the material originating from tumor cells and, in the process, determine tumor purity of a sample (i.e., infer what fraction of the DNA in the sample experienced somatic mutations). Ultimately, however, we are interested in pursuing the task more informative and demanding than the mere separation of tumor's genomic material from the normal one - the task of identifying the number and frequencies of clonal/subclonal/polyclonal populations in a heterogeneous mixture.

While state-of-the-art DNA sequencing technology provides unprecedented insight into individual genetic makeup, accurate reconstruction of tumor genomes remains challenging. On the one hand, high-throughput sequencing technologies generate highly accurate but relatively short reads that cannot span long genomic regions separating SNVs or stretch across long structural aberrations; on the other hand, single-molecule sequencing platforms are capable of reading very long fragments but their accuracy remains inferior to high-throughput short-read platforms. The focus of this paper is on development of fast and accurate computational methods for reconstruction and analysis of heterogeneous mixtures of tumor genomes from short sequencing reads. To this end, we rely on copy number aberrations (i.e., duplications and deletions), which are the most frequently used features for distinguishing components of heterogeneous tumor mixtures. Prior work on this topic includes statistical approaches to the tumor mixture reconstruction in $[19,20]$ and the phylogenetics-based technique in [15]. In contrast, we propose a framework that formulates the reconstruction problem as a mix of convex and combinatorial optimization, and develop scalable algorithms for finding accurate solutions.

The remainder of the paper is organized as follows. Section 2 outlines the mathematical model of the data generated by sequencing a heterogeneous tumor mixture and presents a novel algorithmic framework for reconstructing the components and inferring their relative frequencies. In Section 3, we report results of benchmarking tests conducted on realistic synthetic as well as experimental data and show the comparison with state-of-the-art competing techniques. Section 4 concludes the paper and outlines future work.

\section{METHODOLOGY}

\subsection{Model and the Problem Statement}

To formalize the considered problem and outline our approach to solving it, let us assume that the segments of tumor genomes with gains or losses (i.e., genomic intervals either amplified or deleted) have been detected by relying on existing change-point detection techniques. In particular, assume that there are $M$ such intervals and denote them by $\left\{R_{1}, R_{2}, \ldots, R_{M}\right\}$; let $r_{i}, 1 \leq i \leq M$, denote the average sequencing coverage in region $R_{i}$ normalized by the corresponding coverage of a paired normal sample. ${ }^{1}$ Recall that a sample typically contains both normal cells as well as one or more distinct tumor cell populations. The latter include clonal mutations present in all tumor cells but may also include subclonal mutations present only in a subset of tumor cells. The tumor purity of a sample is defined as the fraction of the tumor cells in a sample. Let $K$ denote the total number of genomes in the mixture and let $f_{1}, f_{2}, \ldots, f_{K}$ be their frequencies; here, $f_{1}$ denotes the fraction of genomes coming from normal cells, i.e.,

$$
1-f_{1}=\sum_{i=2}^{K} f_{i}
$$

is the tumor purity. Finally, let $l_{i j}, 1 \leq i \leq M, 1 \leq j \leq K$, be the copy number of the $j^{t h}$ genome in the $i^{t h}$ region $R_{i}$. Then the normalized sequencing coverage across the $M$ regions can be expressed as

$$
\mathbf{r}=\mathbf{L f},
$$

where $\mathbf{r}=\left[\begin{array}{llll}r_{1} & r_{2} & \ldots & r_{M}\end{array}\right]^{T}, \mathbf{f}=\left[\begin{array}{llll}f_{1} & f_{2} & \ldots & f_{K}\end{array}\right]^{T}$, and matrix $\mathbf{L}$ composed of integer-valued entries is defined as

$$
\mathbf{L}=\left[\begin{array}{cccc}
l_{1,1} & l_{1,2} & \ldots & l_{1, K} \\
l_{2,1} & l_{2,2} & \ldots & l_{2, K} \\
\vdots & \vdots & \ddots & \vdots \\
l_{M, 1} & l_{M, 2} & \ldots & l_{M, K}
\end{array}\right]
$$

The nominal value of $l_{i j}$, i.e., its value in the absence of copy number aberrations in the $j^{\text {th }}$ genome, is 2 (indicating two chromosomes). We assume that the largest number of duplications is $l_{\text {max }}$, that is, $l_{i j} \leq l_{\text {max }}$; it is desirable that the algorithms for learning copy numbers scale gracefully with $l_{\text {max }}$, thus remaining practically feasible even in the presence of large gains.

The problem we are interested in solving can now be stated as follows: Given sequencing coverage $r_{1}, r_{2}, \ldots, r_{M}$ in $M$ genomic regions, find the frequencies of $K$ genomes in a mixture and the matrix $\mathbf{L}$ that specifies copy number aberrations (i.e., gains and losses). Note that the coverage exhibits uncertainties which follow a Poisson distribution. One can therefore formulate the search for $\{\mathbf{f}, \mathbf{L}\}$ as a maximization of an appropriately formed likelihood function. Even at moderate coverage of $\sim 40-50 \mathrm{X}$, typically exceeded in cancer sequencing projects, the Poisson distribution can be approximated by a Gaussian distribution and the maximum-likelihood inference problem leads to constrained optimization of the form

$$
\begin{gathered}
\min _{\mathbf{f}, \mathbf{L}}\|\mathbf{r}-\mathbf{f L}\|_{2}^{2} \\
\text { subject to } \mathbf{f}^{T} \mathbf{1}=1,0 \leq f_{i} \leq 1, \\
0 \leq l_{i j} \leq l_{\max },
\end{gathered}
$$

\footnotetext{
${ }^{1}$ A paired normal sample is sequenced in parallel to sequencing a tumor mixture. Note that we summarize the data in terms of sequencing coverage rather than the more natural number of reads that map to intervals $R_{i}$ solely for the notational simplicity. Rephrasing the model in terms of the number of reads is trivial although expressions end up being somewhat more cumbersome.
} 
where 1 is the column vector of all ones and $l_{i, 1}=2$ for all $i$. The final constraint imposes that the first column of $\mathbf{L}$ corresponds to the normal genome.

\subsection{Proposed Algorithm}

The mixed-integer optimization problem (1) is challenging; we propose to solve it via an alternating minimization procedure in which we keep one of the unknown objects ( $f$ or L) fixed and solve (1) to find the other; the procedure is repeated in an alternating fashion until convergence. In an attempt to escape local minima, we repeat the optimization procedure with different initializations. Due to the mixed nature of optimization (1) $\left(f_{i}\right.$ are real-valued, $l_{i j}$ are integer-valued), the two steps in the alternating optimization procedure require different approaches. We elaborate on them next.

Determining $\mathrm{f}$ given $\mathrm{L}$. In step $t$ of the alternating minimization, one needs to solve convex optimization problem of the form

$$
\min _{\mathbf{f}}\left\|\mathbf{r}-\mathbf{f L}_{t-1}\right\|_{2}^{2}, \quad \text { such that } \mathbf{f}^{T} \mathbf{1}=1,
$$

where $\mathbf{L}_{t-1}$ is determined in step $t-1$ of the procedure. Geometrically, this optimization is essentially concerned with finding the projection of $\mathbf{L}^{T}\left(\mathbf{L L}^{T}\right)^{-1} \mathbf{r}$ onto the probability simplex; $\mathbf{L}^{T}\left(\mathbf{L L}^{T}\right)^{-1} \mathbf{r}$ is the unconstrained solution of the least squares problem in (2). A number of solutions to (2) exist, including the computationally efficient algorithm in [31].

This optimization can be accomplished in a single step, i.e., given $\mathbf{r}$ and $\mathbf{L}_{t-1}$, we can find the optimal $\mathbf{f}$ in one step.

Determining $\mathrm{L}$ given $\mathrm{f}$. Although matrix L has integer-valued entries, it can be found in a computationally efficient manner. First, note that

$$
\min _{\mathbf{L}}\left\|\mathbf{r}-\mathbf{f}_{t} \mathbf{L}\right\|^{2}, \quad \text { such that } 0 \leq l_{i j} \leq l_{\max },
$$

may be decoupled into $M$ subproblems, one for each genomic region $R_{i}$. In particular, one can replace (3) by $M$ optimizations of the form

$$
\min _{l_{1, j}, l_{2, j}, \ldots, l_{K, j}}\left(r_{i}-\sum_{k=1}^{K} f_{t, k} l_{i, k}\right)^{2},
$$

for $i=1,2, \ldots, M$. Optimization (4) is reminiscent of the knapsack [4] and subset sum [25] problems yet is distinct (e.g., unlike in the knapsack problem, the quadratic form of the objective allows selection of $\left\{l_{i, 1}, l_{i, 2}, \ldots, l_{i, K}\right\}$ such that $\sum_{k=1}^{K} f_{k} l_{i, k}$ exceeds $\left.r_{i}\right)$.

The overall procedure, summarized as Algorithm 1, alternates between finding the optimal $f$ and $\mathbf{L}$. To enable a rapid search over the entire space of solutions, $f$ is assigned a random value at the start of each iteration. The maximum number of iterations is a userdefined parameter; we set it to $N=10^{5}$ although approximately equally accurate results are achieved even with significantly fewer iterations. Better results are observed with larger $N$, especially for larger $M$ or $K$, at the expense of longer run times. In each iteration, the projection on the probability simplex takes $O(K \log K)$ operations and the branch-and-bound search has a worst case complexity of $O\left(M l_{\text {max }}{ }^{K}\right)$. However, the latter is an extremely loose upper bound since the pruning of the search space according via branchand-bound dramatically reduces the complexity. This is reflected in the experimental results reported in Section 3.

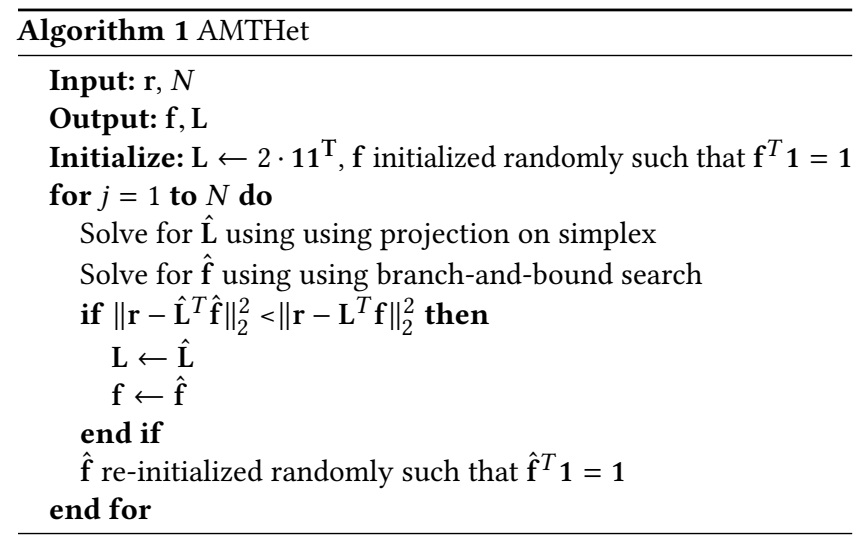

\section{RESULTS}

To evaluate the performance of our algorithm, we compared it with two existing methods for estimation of tumor purity and reconstruction of subclones, THetA [19] and MixClone [13]. Both of these algorithms require a sequence of intervals associated with distinctive copy number variations as the input. THetA assumes a statistical model for the read generation and uses the paired normal sample to determine a weight vector, $\mathbf{w}$ for the model. It optimizes the maximum-likelihood criterion via an exhaustive search over $\mathbf{L}$; for $K=2$, there is a polynomial-time algorithm that finds the solution. MixClone utilizes information on SNPs within the aforementioned intervals in its model formulation. Inference of copy number and tumor fractions is performed on each segment via the expectation-maximization procedure. The performance of different methods was compared on both synthetic and experimental data.

\subsection{Simulated data}

The synthetic data was generated using the same process as SCNVSim [23]. SNCVSim is a simulator which synthesizes realistic cancer genomes with structural variants (SVs) and copy number aberrations (CNAs). To generate the data for benchmarking our proposed algorithm with THetA and MixClone, we used the default parameters of SCNVSim.

In particular, segment lengths were drawn from an exponential distribution with mean $1 \mathrm{Mb}$. All copy numbers were set to 2 as we are assuming diploid genomes. The copy number in each interval was then switched either to $\min \left\{\operatorname{round}(x), l_{\max }\right\}$ or 1 , each with probability 0.05 , where $x$ was drawn from an exponential distribution with mean 3 ; the choice of the parameter of the exponential distribution is made to refrain from having unrealistically large CNAs. The probability used to generate copy numbers is reflective of the default structural variation rate that is used by SCNVSim. Hence, the simulated cancer genomes exhibit highly sparse CNAs most intervals do not have any CNAs in them. The outlined process is repeatedly used to construct tumor genomes until $K-1$ distinct tumor genomes are generated.

In addition to CNAs, MixClone also utilizes information on SNPs within each interval. Following SCNVSim, the heterozygous SNP positions are generated by increments drawn from an exponential distribution with mean $3 \mathrm{~kb}$, i.e., there is, on average, a heterozygous SNP every 1000 bases. This stems from the consideration that the 


\begin{tabular}{|c|c|c|c|c|c|c|c|c|c|}
\hline \multirow{2}{*}{$\mathbf{M}$} & \multicolumn{3}{|c|}{ Purity error } & \multicolumn{3}{c|}{ Tumor fraction error } & \multicolumn{3}{c|}{ CNA error } \\
\cline { 2 - 11 } & THetA & MixClone & AMTHet & THetA & MixClone & AMTHet & THetA & MixClone & AMTHet \\
\hline 6 & 0.166 & 0.243 & 0.013 & 0.234 & 0.506 & 0.018 & 0.156 & 0.366 & 0.0 \\
\hline 8 & 0.135 & 0.328 & 0.049 & 0.191 & 0.618 & 0.07 & 0.131 & 0.301 & 0.013 \\
\hline 10 & 0.057 & 0.466 & 0.037 & 0.08 & 0.701 & 0.053 & 0.032 & 0.248 & 0.0 \\
\hline 12 & 0.065 & 0.334 & 0.02 & 0.091 & 0.598 & 0.028 & 0.008 & 0.217 & 0.0 \\
\hline
\end{tabular}

Table 1: Performance of AMTHet, THetA and MixClone on synthetic data containing a normal and tumor population $(\mathrm{K}=2)$. Maximum copy number is set to 3. AMTHet outperforms other considered algorithms on all metrics.

mean difference between SNP positions is 1000 bases and each SNP postion can be homozygous in either allele or heterozygous. We assumed an equal probability of each of these allelic configurations, thereby giving a heterozygous SNP every $3 \mathrm{~kb}$ on average. The BAllele Frequency for each SNP is randomly set between 0.4 and 0.6 as MixClone uses heterozygous SNP sites for the inference. The variation from 0.5 is to account for sequencing errors in these SNP positions. Finally, the noise model used is identical to that in [19]; the number of reads of length $L_{r}$ from an interval of length $\mathrm{L}_{i}$ is $(1+\phi) \frac{L_{i} c}{L_{r}}$ where $\phi \sim \mathcal{N}(0,0.02)$ and $c$ is the assumed sequencing coverage. Since we have a paired normal sample, for the purpose of benchmarking the choice of $c$ can be arbitrary; we used $c=30$ to generate our synthetic data.

\subsubsection{Error metrics.}

The metrics used to compare the performance of algorithms under consideration are listed below. $\hat{\mathbf{f}}$ and $\hat{\mathbf{L}}$ are the inferred vales of $\mathbf{f}$ and $\mathbf{L}$.

- Purity error. The difference between true and inferred tumor purity, i.e., $\left\|f_{1}-\hat{f}_{1}\right\|_{2}$.

- Tumor fraction error. The difference between true and inferred population frequencies, i.e., $\|\mathbf{f}-\hat{\mathbf{f}}\|_{2}$. This metric is reflective of an algorithm's performance in the settings where there are more than 2 clonal or subclonal populations present (i.e., $K \geq 2$ ); note that the purity error metric does not penalize errors in inferring incorrect proportions of clonal populations.

- CNA error: The average error per entry in the inferred copy number matrix, i.e., $\frac{1}{M(K-1)}\|\mathbf{L}-\hat{\mathbf{L}}\|_{2}$. $\|\mathbf{L}\|_{2}$ refers to the $\ell_{2}$ norm of the vector formed by all the entries in $\mathrm{L}$.

We generated 10 synthetic datasets for each setting of parameters, i.e., for each $\left(M, K, l_{\max }\right)$; the results are averaged to compute means of the above metrics.

\subsubsection{Single tumor population $(\mathrm{K}=2)$.}

Table 1 shows the results for the simplest setting where there is only one tumor population with a maximum copy number $l_{\max }=$ 3. The most striking aspect of the results is the near-zero CNA reconstruction error for AMTHet. The purity and tumor fraction errors achieved by AMTHet are also significantly better than those of its competitors. The accuracy of THetA and MixClone improve as $M$ grows but MixClone exhibits very high errors in terms of metrics characterizing accuracy of inferring population frequencies $f$. A further inspection revealed that MixClone inferred trace amounts of a second tumor cluster. This is likely a consequence of the fact that
MixClone (a) does not assume the presence of a normal population, and. 3 (b) Mqustipte turmstrain the thumor fractions $\mathrm{f}$ to sum to 1 .

The next set of benchmarking tests investigates the performance of different schemes in settings where the number of tumor populations $K$ is greater than 2; the number of genomic regions $M$ is varied. Table 2 shows the results for $K=3$ populations with the maximum copy number $l_{\max }=3$. As evident from the table, AMTHet outperforms MixClone and THetA on every error metric. THetA's performance is adversely affected in this setting since the software hard-codes the assumption that in each genomic interval all tumor genomes can only experience either copy number amplification or a loss. This assumption allows THetA to restrict the space being searched in pursuit of $\mathrm{L}$, which is desirable since the algorithm employs an exhaustive search. Despite this restriction, THetA takes more than 24 hours to run for each set of simulated data when $M=12$; hence the error metrics could not be computed for that combination of parameters $(M, K)$.

The results in Table 2 demonstrate that in the considered settings, AMTHet is more adept at estimating tumor purity than MixClone and achieves an error of under $10 \%$ for all values of $M$. Moreover, AMTHet maintains the performance advantage when it comes to estimating tumor fractions - in particular, its accuracy is at least $50 \%$ higher than that of the competing methods for all $M$. This improvement is further paralleled by an improvement in the accuracy of estimating copy numbers (i.e., L), where AMTHet achieves consistently lower error across the board.

Finally, we report the purity and tumor fraction error rates of different methods for $2 \leq K \leq 5$ graphically in Fig. 2 (a) and (b). Fig. 2(c) compares their runtimes; as pointed out in Section 2, the branchand-bound step in AMTHet has a much lower complexity than what is suggested by the worst-case scenario analysis, $O\left(M l_{\text {max }}{ }^{K}\right)$, due to efficient pruning of the search space. All the algorithms were run on a Windows laptop with a $1.60 \mathrm{GHz}$ Intel Core i5 processor and 8 GM of DDR4 RAM.

\subsection{Breast cancer sequencing data}

To demonstrate the performance of AMTHet on experimental data, we used it to analyze the set comprising paired-end Illumina reads obtained by sequencing genome PD4120a and its matched sample [18]. As elaborated in [19], the data is originally from the European Genome-phenome Archive (accession number: EGAD00001000138). The sample is sequenced at a coverage of $188 \times$ and BIC-Seq is used to segment the 22 chromosomes into intervals based on read depth. Only the intervals longer than $50 \mathrm{~kb}$ were considered. The 


\begin{tabular}{|c|c|c|c|c|c|c|c|c|c|}
\hline \multirow{2}{*}{$\mathbf{M}$} & \multicolumn{3}{|c|}{ Purity error } & \multicolumn{3}{c|}{ Tumor fraction error } & \multicolumn{3}{c|}{ CNA error } \\
\cline { 2 - 10 } & THetA & MixClone & AMTHet & THetA & MixClone & AMTHet & THetA & MixClone & AMTHet \\
\hline 6 & 0.495 & 0.104 & 0.076 & 0.626 & 0.228 & 0.131 & 0.331 & 0.227 & 0.124 \\
\hline 8 & 0.544 & 0.236 & 0.035 & 0.678 & 0.363 & 0.13 & 0.276 & 0.186 & 0.139 \\
\hline 10 & 0.527 & 0.141 & 0.089 & 0.672 & 0.289 & 0.143 & 0.248 & 0.15 & 0.135 \\
\hline 12 & $\times$ & 0.139 & 0.103 & $\times$ & 0.252 & 0.226 & $\times$ & 0.135 & 0.096 \\
\hline
\end{tabular}

Table 2: Performance of AMTHet, THetA and MixClone on synthetic data containing three populations $(\mathrm{K}=3)$, with $\mathrm{l}_{\max }=3$. AMTHet achieves higher accuracy than other algorithms in terms of all metrics.

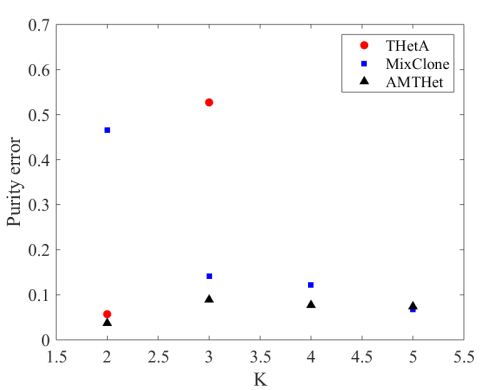

(a)

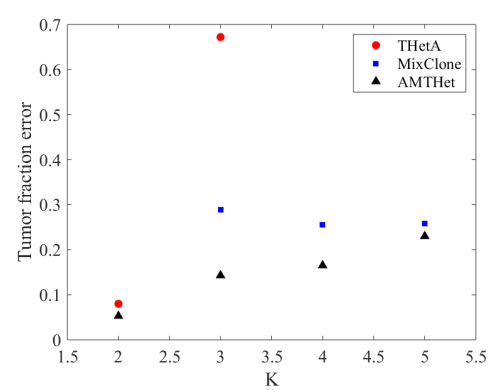

(b)

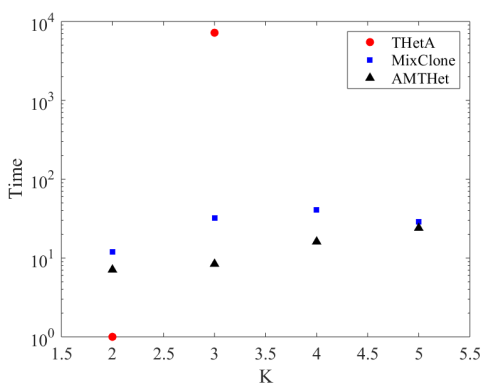

(c)

Figure 2: (a) Average purity error, (b) tumor fraction inference error and (c) run time of THetA, MixClone and AMTHet evaluated over $M=10$ segments. THetA could only be executed for $K=2,3$ as it involves an exhaustive search over $L$, so it is computationally intractable for larger $K$.

segmented intervals along with the number of aligned reads in each interval were kindly provided by the authors of [19]; this pre-processed data does not contain SNP information needed for testing the performance of MixClone.

Sequencing coverage of the breast cancer sample and its matched normal sample generally differ from each other. To account for this mismatch, we determined a multiplicative factor using a genomic region known not to contain any CNAs. For the particular data being analyzed, the first interval was suitable for the task of determining the multiplicative factor. Denoting the number of reads aligned to the first interval in the tumor and normal sample as $r_{t 1}$ and $r_{n 1}$, respectively, we multiplied the read counts for all the intervals in the normal sample by $\frac{r_{t 1}}{r_{n 1}}$. When THetA and AMTHet were executed in the $K=2$ scenario, they estimated the tumor purity to be $65.5 \%$ and $65 \%$, respectively; their results are very close, albeit slightly lower than the estimate of $70 \%$ in [18].

Among CNAs annotated in [18], both THetA and AMTHet succeed in inferring the amplification in 1q. Similarly, both algorithms discovered the deletions in 1p, 4q, 6p, 13, 16q and 22q. AMTHet discovered the deletion in $21 \mathrm{p}$ as well, which is documented in [18]. Moreover, inferrenced copy numbers are more contiguous in AMTHet's result than THetA's; THetA appears to infer spurious amplifications in intervals 28 and 248.

\section{CONCLUSIONS AND FUTURE WORK}

In this paper, we studied the problem of reconstructing genomic components of a heterogenous tumor mixture analyzed by highthroughput DNA sequencing data. In particular, we developed an algorithmic framework that relies on detected locations of copy number aberrations to quantify gains and losses of genomic material in those regions, and ultimately determines the number and frequencies of populations in a clonal mixture. The algorithm, AMTHet, considers the reconstruction as a mixed-integer optimization problem and solves it via a combination of convex optimization and branch-and-bound search. AMTHet was tested on realistic synthetic as well as experimental data and shown to outperform state-of-the art competing methods in a variety of scenarios. Ongoing and future work includes extension of the AMTHet framework so that it allows incorporation of SNP information and further tests on experimental datasets.

\section{ACKNOWLEDGMENTS}

This work was funded in part by the NSF grant CCF 1618427.

\section{REFERENCES}

[1] Ludmil B. Alexandrov, Serena Nik-Zainal, David C. Wedge, Samuel A. J. R. Aparicio, Sam Behjati, Andrew V. Biankin, Graham R. Bignell, Niccolò Bolli, Ake Borg, Anne-Lise Børresen-Dale, Sandrine Boyault, Birgit Burkhardt, Adam P. Butler, Carlos Caldas, Helen R. Davies, Christine Desmedt, Roland Eils, Jórunn Erla Eyfjörd, John A. Foekens, Mel Greaves, Fumie Hosoda, Barbara Hutter, Tomislav Ilicic, Sandrine Imbeaud, Marcin Imielinski, Natalie Jäger, David T. W. Jones, David Jones, Stian Knappskog, Marcel Kool, Sunil R. Lakhani, Carlos López-Otín, Sancha Martin, Nikhil C. Munshi, Hiromi Nakamura, Paul A. Northcott, Marina Pajic, Elli Papaemmanuil, Angelo Paradiso, John V. Pearson, Xose S. Puente, Keiran Raine, Manasa Ramakrishna, Andrea L. Richardson, Julia Richter, Philip Rosenstiel, Matthias Schlesner, Ton N. Schumacher, Paul N. Span, Jon W. Teague, Yasushi Totoki, Andrew N. J. Tutt, Rafael Valdés-Mas, Marit M. van Buuren, Laura 
van 't Veer, Anne Vincent-Salomon, Nicola Waddell, Lucy R. Yates, Australian Pancreatic Cancer Genome Initiative, ICGC Breast Cancer Consortium, ICGC MMML Seq Consortium, ICGC PedBrain, Jessica Zucman-Rossi, P. Andrew Futreal, Ultan McDermott, Peter Lichter, Matthew Meyerson, Sean M. Grimmond, Reiner Siebert, Elías Campo, Tatsuhiro Shibata, Stefan M. Pfister, Peter J. Campbell, and Michael R. Stratton. 2013. Signatures of mutational processes in human cancer Nature 500 (14 08 2013), 415 EP -. http://dx.doi.org/10.1038/nature12477

[2] J. Kenneth Baillie, Mark W. Barnett, Kyle R. Upton, Daniel J. Gerhardt, Todd A Richmond, Fioravante De Sapio, Paul M. Brennan, Patrizia Rizzu, Sarah Smith, Mark Fell, Richard T. Talbot, Stefano Gustincich, Thomas C. Freeman, John S Mattick, David A. Hume, Peter Heutink, Piero Carninci, Jeffrey A. Jeddeloh, and Geoffrey J. Faulkner. 2011. Somatic retrotransposition alters the genetic landscape of the human brain. Nature 479 (30 10 2011), $534 \mathrm{EP}$-. http://dx.doi.org/10.1038/ nature 10531

[3] Niko Beerenwinkel, Roland F. Schwarz, Moritz Gerstung, and Florian Markowetz 2015. Cancer Evolution: Mathematical Models and Computational Inference. Systematic Biology 64, 1 (2015), e1-e25. https://doi.org/10.1093/sysbio/syu081

[4] George B Dantzig. 1957. Discrete-variable extremum problems. Operations research 5, 2 (1957), 266-288 0030-364X

[5] Jin-Tang Dong. 2001. Chromosomal Deletions and Tumor Suppressor Genes in Prostate Cancer. Cancer and Metastasis Reviews 20, 3 (2001), 173-193. https //doi.org/10.1023/A:1015575125780

[6] Mohammed El-Kebir, Layla Oesper, Hannah Acheson-Field, and Benjamin J Raphael. 2015. Reconstruction of clonal trees and tumor composition from multi-sample sequencing data. Bioinformatics 31, 12 (2015), i62-i70. https: //doi.org/10.1093/bioinformatics/btv261

[7] Edward D Esplin, Ling Oei, and Michael P Snyder. 2014. Personalized se quencing and the future of medicine: discovery, diagnosis and defeat of disease. Pharmacogenomics 15, 14 (2014), 1771-1790. https://doi.org/10.2217/pgs.14.117 arXiv:https://doi.org/10.2217/pgs.14.117 PMID: 25493570

[8] Manuel L Gonzalez-Garay. 2014. The road from next-generation sequencing to personalized medicine. Personalized Medicine 11, 5 (2014), 523-544. https://doi. org/10.2217/pme.14.34 arXiv:https://doi.org/10.2217/pme.14.34 PMID: 26000024

[9] Ravi G Gupta and Robert A Somer. 2017. Intra-tumor Heterogeneity: Novel Approaches for Resolving Genomic Architecture and Clonal Evolution. Molecular Cancer Research (2017). https://doi.org/10.1158/1541-7786. MCR-17-0070 arXiv:http://mcr.aacrjournals.org/content/early/2017/06/08/15417786.MCR-17-0070.full.pdf

[10] Wei Jiao, Shankar Vembu, Amit G. Deshwar, Lincoln Stein, and Quaid Morris. 2014 Inferring clonal evolution of tumors from single nucleotide somatic mutations. BMC Bioinformatics 15, 1 (2014), 35. https://doi.org/10.1186/1471-2105-15-35

[11] Pamela S Larson, Benjamin L Schlechter, Antonio de las Morenas, Judy E Garber, L Adrienne Cupples, and Carol L Rosenberg. 2005. Allele imbalance, or loss of heterozygosity, in normal breast epithelium of sporadic breast cancer cases and BRCA1 gene mutation carriers is increased compared with reduction mammoplasty tissues. Journal of clinical oncology 23, 34 (2005), 8613-8619.

[12] Michael S. Lawrence, Petar Stojanov, Paz Polak, Gregory V. Kryukov, Kristian Cibulskis, Andrey Sivachenko, Scott L. Carter, Chip Stewart, Craig H. Mermel, Steven A. Roberts, Adam Kiezun, Peter S. Hammerman, Aaron McKenna, Yotam Drier, Lihua Zou, Alex H. Ramos, Trevor J. Pugh, Nicolas Stransky, Elena Helman, Jaegil Kim, Carrie Sougnez, Lauren Ambrogio, Elizabeth Nickerson, Erica Shefler, Maria L. Cortés, Daniel Auclair, Gordon Saksena, Douglas Voet, Michael Noble, Daniel DiCara, Pei Lin, Lee Lichtenstein, David I. Heiman, Timothy Fennell, Marcin Imielinski, Bryan Hernandez, Eran Hodis, Sylvan Baca, Austin M. Dulak, Jens Lohr, Dan-Avi Landau, Catherine J. Wu, Jorge Melendez-Zajgla, Alfredo Hidalgo-Miranda, Amnon Koren, Steven A. McCarroll, Jaume Mora, Ryan S. Lee, Brian Crompton, Robert Onofrio, Melissa Parkin, Wendy Winckler, Kristin Ardlie, Stacey B. Gabriel, Charles W. M. Roberts, Jaclyn A. Biegel, Kimberly Stegmaier, Adam J. Bass, Levi A. Garraway, Matthew Meyerson, Todd R. Golub, Dmitry A. Gordenin, Shamil Sunyaev, Eric S. Lander, and Gad Getz. 2013. Mutational heterogeneity in cancer and the search for new cancer-associated genes. Nature 499 (16 06 2013), $214 \mathrm{EP}$-. http://dx.doi.org/10.1038/nature12213

[13] Yi Li and Xiaohui Xie. 2015. Mixclone: a mixture model for inferring tumor subclonal populations. In BMC genomics, Vol. 16. BioMed Central, S1.

[14] W W Lockwood, R Chari, B P Coe, L Girard, C MacAulay, S Lam, A F Gazdar, J D Minna, and W L Lam. 2008. DNA amplification is a ubiquitous mechanism of oncogene activation in lung and other cancers. Oncogene 27 (07 04 2008), 4615 EP -. http://dx.doi.org/10.1038/onc.2008.98

[15] Salem Malikic, Andrew W. McPherson, Nilgun Donmez, and Cenk S. Sahinalp. 2015. Clonality inference in multiple tumor samples using phylogeny. Bioinformatics 31, 9 (2015), 1349-1356. https://doi.org/10.1093/bioinformatics/btv003
[16] Iñigo Martincorena and Peter J. Campbell. 2015. Somatic mutation in cancer and normal cells. Science 349, 6255 (2015), 1483-1489. https://doi.org/10.1126/science. aab4082 arXiv:http://science.sciencemag.org/content/349/6255/1483.full.pdf

[17] Iñigo Martincorena, Amit Roshan, Moritz Gerstung, Peter Ellis, Peter Van Loo, Stuart McLaren, David C Wedge, Anthony Fullam, Ludmil B Alexandrov, and Jose M Tubio. 2015. High burden and pervasive positive selection of somatic mutations in normal human skin. Science 348, 6237 (2015), 880-886 0036-8075.

[18] Serena Nik-Zainal, Peter Van Loo, David C Wedge, Ludmil B Alexandrov, Christopher D Greenman, King Wai Lau, Keiran Raine, David Jones, John Marshall, and Manasa Ramakrishna. 2012. The life history of 21 breast cancers. Cell 149, 5 (2012), 994-1007 0092-8674

[19] Layla Oesper, Ahmad Mahmoody, and Benjamin J. Raphael. 2013. THetA: inferring intra-tumor heterogeneity from high-throughput DNA sequencing data. Genome Biology 14, 7 (2013), R80. https://doi.org/10.1186/gb-2013-14-7-r80

[20] Layla Oesper, Anna Ritz, Sarah J Aerni, Ryan Drebin, and Benjamin J Raphael. 2012. Reconstructing cancer genomes from paired-end sequencing data. BMC Bioinformatics 13, Suppl 6 (2012), S10-S10. https://doi.org/10.1186/ 1471-2105-13-S6-S10

[21] Bradley A. Perkins, C. Thomas Caskey, Pamila Brar, Eric Dec, David S. Karow, Andrew M. Kahn, Ying-Chen Claire Hou, Naisha Shah, Debbie Boeldt, Erin Coughlin, Gabby Hands, Victor Lavrenko, James Yu, Andrea Procko, Julia Appis, Anders M. Dale, Lining Guo, Thomas J. Jönsson, Bryan M. Wittmann, Istvan Bartha, Smriti Ramakrishnan, Axel Bernal, James B. Brewer, Suzanne Brewerton, William H. Biggs, Yaron Turpaz, and J. Craig Venter. 2018. Precision medicine screening using whole-genome sequencing and advanced imaging to identify disease risk in adults. Proceedings of the National Academy of Sciences 115, 14 (2018), 3686-3691. https://doi.org/10.1073/pnas.1706096114 arXiv:http://www.pnas.org/content/115/14/3686.full.pdf

[22] Victoria Popic, Raheleh Salari, Iman Hajirasouliha, Dorna Kashef-Haghighi, Robert B West, and Serafim Batzoglou. 2015. Fast and scalable inference of multi-sample cancer lineages. Genome Biology 16, 1 (2015), 91. https: //doi.org/10.1186/s13059-015-0647-8

[23] Maochun Qin, Biao Liu, Jeffrey M Conroy, Carl D Morrison, Qiang Hu, Yubo Cheng, Mitsuko Murakami, Adekunle O Odunsi, Candace S Johnson, Lei Wei, et al. 2015. SCNVSim: somatic copy number variation and structure variation simulator. BMC bioinformatics 16, 1 (2015), 66.

[24] Benjamin J. Raphael, Jason R. Dobson, Layla Oesper, and Fabio Vandin. 2014. Identifying driver mutations in sequenced cancer genomes: computational approaches to enable precision medicine. Genome Medicine 6, 1 (2014), 5. https://doi.org/10.1186/gm524

[25] Claus-Peter Schnorr and Martin Euchner. 1994. Lattice basis reduction: Improved practical algorithms and solving subset sum problems. Mathematical programming 66, 1-3 (1994), 181-199.

[26] Ronglai Shen and Venkatraman E. Seshan. 2016. FACETS: allele-specific copy number and clonal heterogeneity analysis tool for high-throughput DNA sequencing. Nucleic Acids Research 44, 16 (2016), e131. https://doi.org/10.1093/nar/gkw520

[27] Giorgio Stanta and Serena Bonin. 2018. Overview on Clinical Relevance of IntraTumor Heterogeneity. Frontiers in Medicine 5 (2018), 85. https://doi.org/10.3389/ fmed.2018.00085

[28] Philip J. Stephens, David J. McBride, Meng-Lay Lin, Ignacio Varela, Erin D. Pleasance, Jared T. Simpson, Lucy A. Stebbings, Catherine Leroy, Sarah Edkins, Laura J. Mudie, Chris D. Greenman, Mingming Jia, Calli Latimer, Jon W. Teague, King Wai Lau, John Burton, Michael A. Quail, Harold Swerdlow, Carol Churcher, Rachael Natrajan, Anieta M. Sieuwerts, John W. M. Martens, Daniel P. Silver, Anita Langerød, Hege E. G. Russnes, John A. Foekens, Jorge S. Reis-Filho, Laura van 't Veer, Andrea L. Richardson, Anne-Lise Børresen-Dale, Peter J. Campbell, P. Andrew Futreal, and Michael R. Stratton. 2009. Complex landscapes of somatic rearrangement in human breast cancer genomes. Nature 462 (24 12 2009), 1005 EP -. http://dx.doi.org/10.1038/nature08645

[29] Xiao-xiao Sun and Qiang Yu. 2015. Intra-tumor heterogeneity of cancer cells and its implications for cancer treatment. Acta Pharmacologica Sinica 36, 10 (10 2015), 1219-1227. https://doi.org/10.1038/aps.2015.92

[30] Bert Vogelstein, Nickolas Papadopoulos, Victor E Velculescu, Shibin Zhou, Luis A Diaz, and Kenneth W Kinzler. 2013. Cancer genome landscapes. Science 339, 6127 (2013), 1546-1558 0036-8075.

[31] Weiran Wang and Miguel A Carreira-Perpinán. 2013. Projection onto the probability simplex: An efficient algorithm with a simple proof, and an application. arXiv preprint arXiv:1309.1541 (2013).

[32] Amy H Yang, Dhruv Kaushal, Stevens K Rehen, Kristin Kriedt, Marcy A Kingsbury, Michael J McConnell, and Jerold Chun. 2003. Chromosome segregation defects contribute to aneuploidy in normal neural progenitor cells. Fournal of Neuroscience 23, 32 (2003), 10454-10462. 